\title{
Propuesta para la creación de un fondo de capital de riesgo para la Universidad Pedagógica y Tecnológica de Colombia sede Sogamoso
}

\author{
Proposal for the creation of a venture capital fund for the pedagogical and \\ technological university of Colombia headquarters Sogamoso \\ Luis Germán Báez Mancera \\ Escuela de Administración de Empresas, Universidad Pedagógica y Tecnológica de Colombia, Sogamoso, \\ Colombia \\ German.baezeuptc.edu.co
}

\begin{abstract}
Resumen- En el presente artículo se abordan los resultados del estudio de investigación de mercados como avance del proyecto de investigación para la creación de un fondo de capital de riesgo para la Universidad Pedagógica y Tecnológica de Colombia sede Sogamoso. Realizado por el grupo de investigación management adscrito al programa de administración de empresas. Que permita apalancar los spin-off universitarios y proyectos de investigación de base tecnológica e innovadores que se originen en los proyectos de investigación realizados por docentes, estudiantes y egresados de la sede.
\end{abstract}

Palabras clave - capital de riesgo, innovación, investigación de mercados, universidad.

Abstract - In this article the results of the market research and progress of the research project for the creation of a venture capital fund for the Pedagogical and Technological University of Colombia at Sogamoso. Made by the research group attached to the program management business administration. Allowing leverage and university spin-off research projects and innovative database technology originating in research projects conducted by faculty, students and graduates of the seat.

\section{INTRODUCCIÓN}

El grupo de investigación management adscrito al programa de administración de empresas se encuentra desarrollando un proyecto de investigación para la creación de un Fondo de Capital de Riesgo para la Universidad Pedagógica y Tecnológica de Colombia sede Sogamoso ya que esta es una necesidad imperante para por esta vía apalancar y consolidar spin-off universitarios, patentes y proyectos de base tecnológica generados por profesores, estudiantes y egresados de los diferentes programas académicos de la sede, que garanticen el desarrollo empresarial a nivel; investigativo, tecnológico, creativo e innovador como elemento o factor esencial del quehacer universitario, que conduzca a dinamizar el crecimiento y fortalecimiento económico de la región y el país, como alternativa preponderante en la necesidad de canalizar recursos financieros hacia los emprendimientos que surjan de los diferentes proyectos de investigación de la UPTC sede Sogamoso.

\section{ANTECEDENTES}

El área de Boston fue quizás la primera región en mostrar un cierto grado de inversión de capital de riesgo de forma organizada. En 1911, la Cámara de Comercio de Boston prestaba asistencia financiera y técnica a nuevas empresas y, en 1940, la Corporación para el desarrollo industrial de Nueva Inglaterra comenzó a ofrecer un tipo similar de asistencia [1]. Boston fue también el hogar de los primeros fondos de capital de riesgo de los EE.UU. La idea del capital de riesgo fue de Flandes Ralph, presidente de la Reserva Federal de Boston, que estaba preocupado por la falta de constitución de nuevas empresas y la incapacidad de los inversores institucionales para financiar los nuevos emprendimientos. Flandes propuso a 
los fondos fiduciarios, que permitirían a los inversionistas institucionales invertir hasta un 5 por ciento de su capital en nuevas empresas [2]. La propuesta fue apoyada por el General Georges Doriot (profesor de la Harvard Business School) y, junto con Carl Compton (presidente del MIT(Instituto Tecnológico de Masachusets)) y algunos líderes empresariales locales, Doriot estableció el American Research and Development (ARD) en 1946. ARD realizando inversiones en empresas jóvenes con base en tecnologías desarrolladas para la Segunda Guerra Mundial, a menudo con estrechos vínculos con Harvard y el MIT. Su primera inversión fue en la corporación ingeniería de alto voltaje, que fue fundada por ingenieros del MIT y que más tarde se convirtió en la primera firma financiada con capital de riesgo listada en la Bolsa de valores de Nueva York. Sin embargo, no todas las inversiones tuvieron éxito, casi la mitad de las ganancias de ARD durante su existencia de 26 años provinieron de sus US $\$ 70.000$ de inversión en la empresa Digital Equipment, en 1957, que había incrementado su valor a 355 millones de dólares en 1971 [3].

En Silicon Valley (San Francisco), otra región con un grupo denso de empresas de base tecnológica, los grupos de capital de riesgo comenzaron a surgir durante la década de 1950 y principios de la década de 1960. La primera firma de capital de riesgo en California Draper \& Gaither, y Andersen fue fundada en 1958, a finales de la década de 1950 se convirtió en un período de auge de creación de más de una docena de empresas de capital de riesgo en Silicon Valley.

\section{CONTENIDO}

\section{A. Capital de riesgo}

El Capital Riesgo (Venture Capital) es una actividad financiera que proporciona recursos a empresas innovadoras de base tecnológica con dificultades para obtener otro tipo de financiación, a medio y largo plazo y sin vocación de permanencia ilimitada. Toma participación en las empresas y les ofrece apoyo y asesoramiento gerencial como valor agregado. Los inversores pueden ser tanto públicos como privados. Su objetivo es, en cualquier caso, obtener beneficios económicos y financieros si se trata de inversionistas privados y regeneración del tejido empresarial si son públicos.

\section{B. Tipo de investigación}

La base de investigación para el desarrollo de este trabajo obedece a la revisión de información, análisis de casos, aplicación de encuestas y experiencias que se han dado en espacios diferentes a la UPTC, para lo cual se requiere conocimiento de proyectos similares. Así la investigación estará demarcada dentro del enfoque mixto donde prevalecerá el análisis cuantitativo reforzado con herramientas del enfoque cualitativo cuando en el desarrollo del proyecto se tenga que acudir a relatos y revelaciones de expertos en la consolidación de Fondos de Capital de Riesgo universitarios.

\section{Estudio de investigación de mercados}

En esta primer etapa de desarrollo del proyecto para la creación de un fondo de capital de riesgo se realiza un estudio de investigación de mercados [4], que permitirá a través de la encuesta aplicada a los docentes de la UPTC sede Sogamoso recabar información relevante respecto de: las necesidades de financiación de los proyectos de investigación de base tecnológica e innovadores que tienen origen en la UPTC sede Sogamoso, en que fase de desarrollo se encuentran los proyectos de investigación que se están desarrollando actualmente, los activos de propiedad intelectual existentes o que se encuentran en desarrollo, si la falta de financiamiento a sido un obstáculo para el desarrollo de sus proyectos de investigación, el tipo de financiación que se piensa utilizar para apalancar los proyectos, si se considera o no importante la creación de un fondo de capital de riesgo para la sede, la preferencia o percepción al riesgo.

D. tamaño de la muestra y método de muestreo

Para la selección de la muestra se utiliza un muestreo aleatorio estratificado con asignación proporcional, en el cual el tamaño de la muestra está definido por:

$\mathrm{n}=\left(\mathrm{NZ}{ }^{2} \mathrm{PQ} /(\mathrm{N}-1) \mathrm{E} 2+\mathrm{Z}^{2} \mathrm{PQ}\right)[5]$

Donde:

$\mathrm{n}=$ Tamaño de la muestra

$\mathrm{N}=$ Tamaño de la población

$\mathrm{P}=$ Probabilidad de ocurrencia de la selección muestral (50\%)

$\mathrm{Q}=$ Probabilidad de ocurrencia de la selección no muestral

$(50 \%)$

$\mathrm{E}=$ Error de muestreo

$\mathrm{Z}=$ Valor crítico de $\mathrm{k}$ de la distribución normal estándar de

1.96, para un nivel de confianza del $95 \%$.

Docentes UPTC sede Sogamoso

La tabla 1. Muestra la distribución de docentes (planta tiempo completo, ocasionales tiempo completo, ocasionales medio tiempo y catedráticos) de la UPTC sede Sogamoso en el primer semestre de 2014. 


\begin{tabular}{|l|c|c|c|c|c|}
\hline PROGRAMA & $\begin{array}{c}\text { PLANTA } \\
\text { T.C }\end{array}$ & $\begin{array}{c}\text { OCASIONALES } \\
\text { T.C }\end{array}$ & $\begin{array}{c}\text { OCASIONALES } \\
\text { M.T }\end{array}$ & CATEDRÁTICOS & TOTAL \\
\hline $\begin{array}{l}\text { INGENIERÍA DE } \\
\text { MINAS }\end{array}$ & 12 & 4 & 0 & 8 & 24 \\
\hline $\begin{array}{l}\text { INGENIERIA } \\
\text { GEOLÓGICA }\end{array}$ & 12 & 3 & 1 & 13 & 29 \\
\hline $\begin{array}{l}\text { ADMINISTRACIÓN } \\
\text { DE EMPRESAS }\end{array}$ & 7 & 8 & 3 & 3 & 21 \\
\hline $\begin{array}{l}\text { INGENIERIA } \\
\text { ELECTRÓNICA }\end{array}$ & 13 & 6 & 0 & 4 & 23 \\
\hline $\begin{array}{l}\text { CONTADURİA } \\
\text { PUBLICA }\end{array}$ & 7 & 13 & 2 & 5 & 27 \\
\hline $\begin{array}{l}\text { INGENIERIA } \\
\text { INDUSTRIAL }\end{array}$ & 13 & 6 & 1 & 7 & 27 \\
\hline $\begin{array}{l}\text { INGFNIFRÍA DF. } \\
\text { SISTEMAS }\end{array}$ & 0 & 6 & 1 & 4 & 11 \\
\hline TOTAL & 64 & 46 & 8 & 44 & 162 \\
\hline
\end{tabular}

Tabla 1: Docentes UPTC sede Sogamoso

En la tabla 2. Se puede evidenciar la vinculación de docentes siendo los docentes de planta tiempo completo los de mayor participación dentro del total ,con el $40 \%$. Seguido de los docentes ocasionales de tiempo completo con un $28 \%$ de participación, los catedráticos representan el 27\%.

\begin{tabular}{|l|c|c|}
\hline VINCULACIÓN & DOCENTES & $\%$ \\
\hline PLANTA TIEMPO COMPLETO & 64 & $40 \%$ \\
\hline OCASIONALES T.C & 46 & $28 \%$ \\
\hline OCASIONALES M.T & 8 & $5 \%$ \\
\hline CATEDRA & 44 & $27 \%$ \\
\hline TOTAL & 162 & $100 \%$ \\
\hline
\end{tabular}

Tabla 2: Vinculación docentes UPTC sede Sogamoso

Tamaño de la muestra

Con un nivel de confianza del 95\% y un error del 5\% y aplicado la fórmula para poblaciones finitas variable cualitativa se obtuvo el resultado mostrado en la tabla 3.

\begin{tabular}{|l}
$\mathrm{Z}=1,96$ \\
$\mathrm{~N}=162$ \\
$\mathrm{P}=0,5$ \\
$\mathrm{Q}=0,5$ \\
$\mathrm{E}=0,05$ \\
$\mathrm{n}=114$
\end{tabular}

Tabla 3: Tamaño de la muestra
Distribución de la muestra

En la selección de la muestra se tuvo en cuenta como población objetivo el total de docentes de la sede y se aplicó un muestreo estratificado, la selección de cada elemento de la muestra se obtuvo mediante el muestreo aleatorio simple como se muestra en la tabla 4.

\begin{tabular}{|l|c|}
\hline \multicolumn{1}{|c|}{ PROGRAMA } & $\begin{array}{c}\text { ELEMENTO EN } \\
\text { LAS MUESTRA }\end{array}$ \\
\hline INGENIERÍA DE MINAS & 17 \\
\hline INGENIERÍA GEOLÓGICA & 20 \\
\hline ADMINISTRACIÓN DE EMPRESAS & 15 \\
\hline INGENIERÍA ELECTRÓNICA & 16 \\
\hline CONTADURÍA PUBLICA & 19 \\
\hline INGENIERÍA INDUSTRIAL & 19 \\
\hline INGENIERÍA DE SISTEMAS & 8 \\
\hline TOTAL MUESTRA & 114 \\
\hline
\end{tabular}

Tabla 4: Distribución de la muestra

\section{ANÁLISIS Y CARACTERÍSTICAS DE LOS ENCUESTADOS}

El $40 \%$ de docentes de la UPTC sede Sogamoso son de planta mientras que el $60 \%$ son docentes ocasionales y catedráticos como se evidencia en la gráfica $I$.

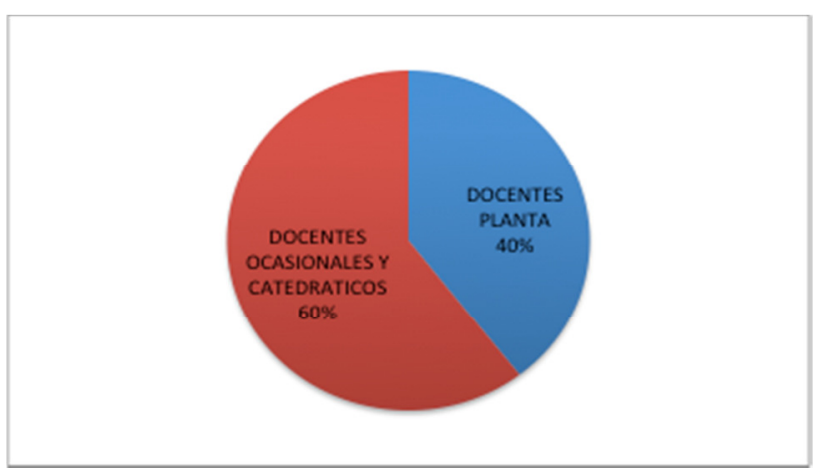

Grafica 1. Tipo de vinculación docentes UPTC- Sogamoso

Pregunta 1.

¿Se encuentra actualmente desarrollando un proyecto de investigación de base tecnológica e innovador?

El 39\% de los docentes de la UPTC-Sogamoso se encuentra actualmente realizando un proyecto de investigación de base tecnológica e innovador y el $61 \%$ manifiesta no estar realizando ningún proyecto, como se muestra en la gráfica 2 . 


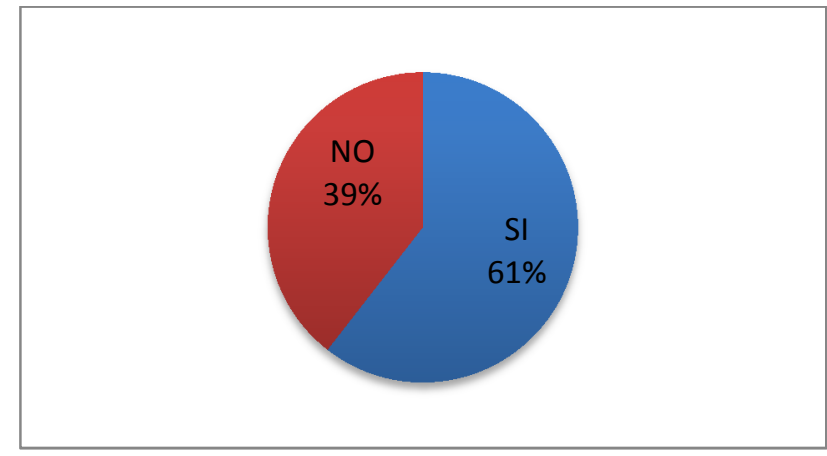

Grafica 2. Se encuentra desarrollando proyectos de investigación

Pregunta 2.

¿En qué etapa de desarrollo se encuentra su proyecto de investigación?

De los docentes que se encuentran actualmente desarrollando un proyecto de investigación de base tecnológica e innovador, Grafica 3. El 38\% está en la etapa de gestación, el $17 \%$ en etapa de inicio, el $33 \%$ en etapa de crecimiento y el $12 \%$ en etapa de consolidación.

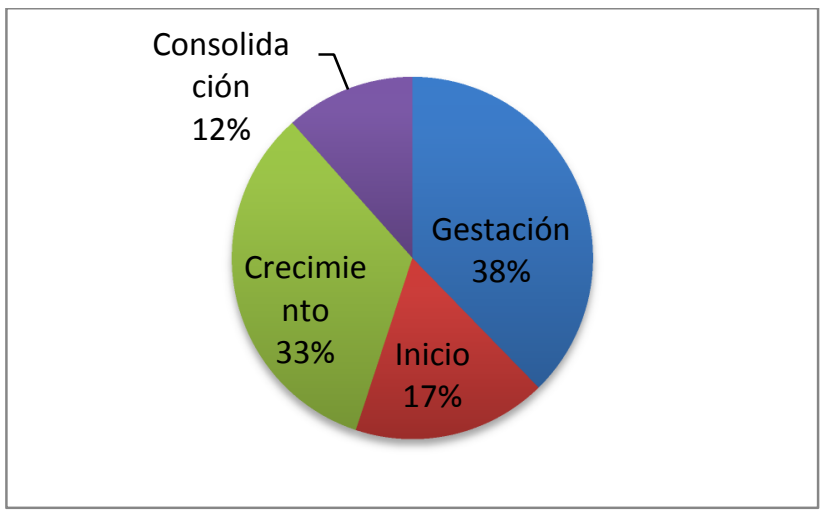

Grafica 3. Etapa de desarrollo proyecto

Pregunta 3.

¿Lidera actualmente la creación de una nueva iniciativa empresarial universitaria (Spin-offs)?

El la gráfica 4, se observa que el $26 \%$ de los docentes de la UPTC-Sogamoso se encuentran liderando actualmente la creación de una nueva iniciativa empresarial universitaria (Spin-offs) mientras que el $74 \%$ no lidera actualmente ninguna iniciativa empresarial universitaria.

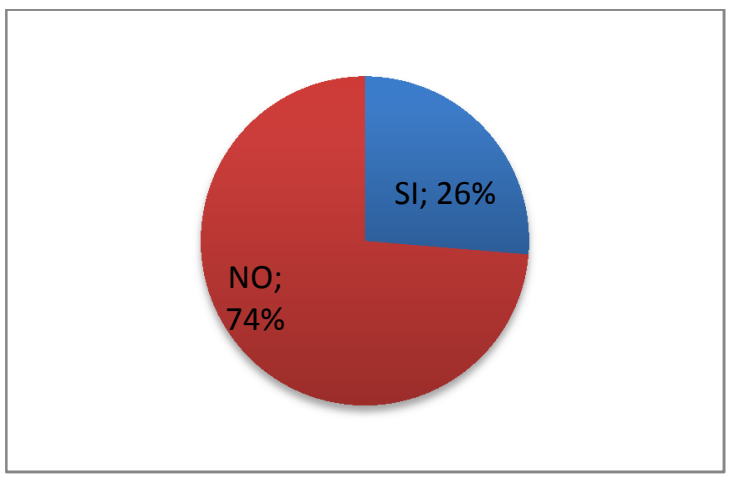

Grafica 4. Creación de Spin-offs

Pregunta 4.

¿Está usted desarrollando o es dueño de activo(s) de propiedad intelectual?

El 7\% de los docentes encuestados es dueño o está desarrollando activos de propiedad intelectual específicamente diseño industrial, el $6 \%$ es propietario de patentes y el $87 \%$ no es propietario ni se encuentra desarrollando actualmente activos de propiedad intelectual, Grafica 5.

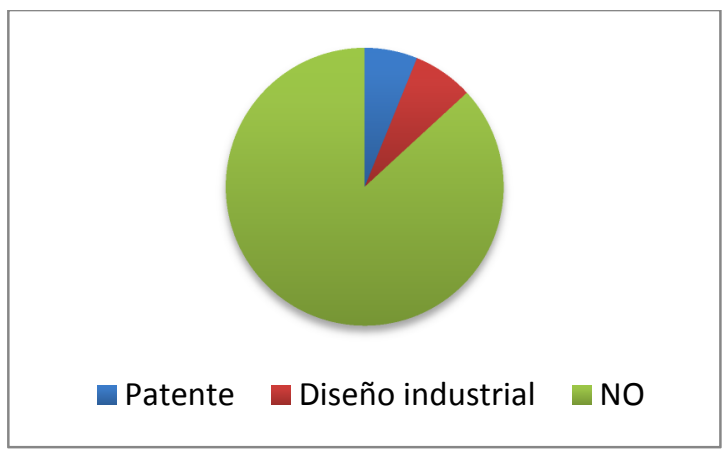

Grafica 5. Activos de propiedad intelectual

Pregunta 5.

¿La falta de financiamiento ha sido un obstáculo para el desarrollo de su idea de negocio, Spin-offs o activo de propiedad intelectual?

Para el $87 \%$ de los docentes la falta de financiamiento ha sido un obstáculo para el desarrollo de su idea de negocio, Spinoffs o activo de propiedad intelectual mientras que para el $13 \%$ esta no ha sido una dificultad, grafica 6 . 


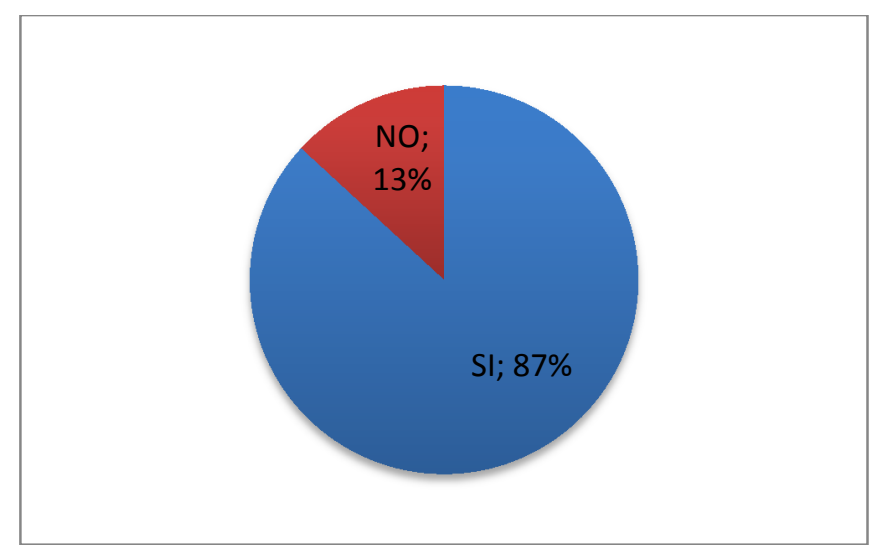

Grafica 6. Falta de financiamiento para el desarrollo de proyectos

Pregunta 6.

¿Qué tipo de financiación piensa utilizar para apalancar el proyecto de investigación, Spin-off o activo de propiedad intelectual?

El capital de riesgo es la fuente de financiación que los docentes de la UPTC-Sogamoso piensan utilizar para apalancar sus proyectos de investigación con un $56 \%$ mientras que el $40 \%$ piensa utilizar los recursos propios para apalancar sus proyectos de investigación y el 4\% manifiesta que utilizara el crédito bancario, Grafica 7.

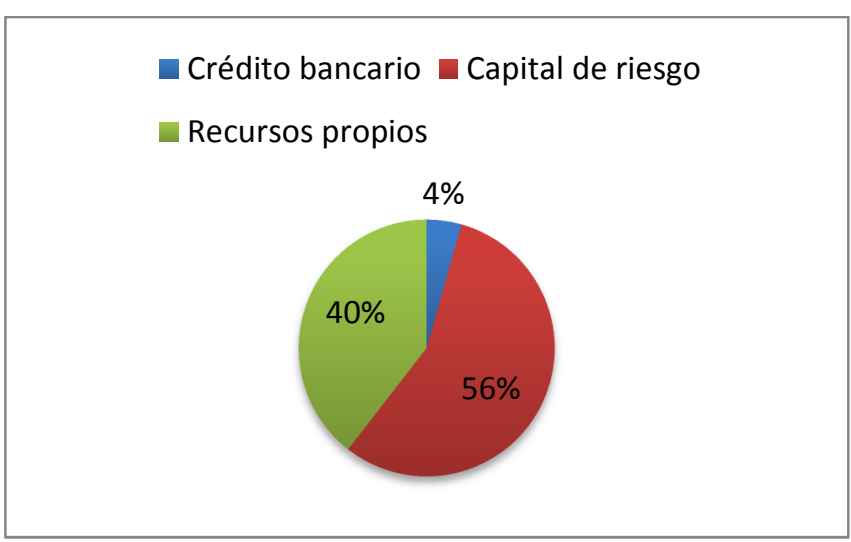

Grafica 7. Tipo de financiación para apalancar proyecto de investigación

Pregunta 7.

¿Considera usted importante la creación de un fondo de capital de riesgo para la UPTC sede Sogamoso?

Para el $92 \%$ de los docentes es importante la creación de un fondo de capital de riesgo para la UPTC-Sogamoso mientras que para el $8 \%$ de los docentes no es importante, Grafica 8.

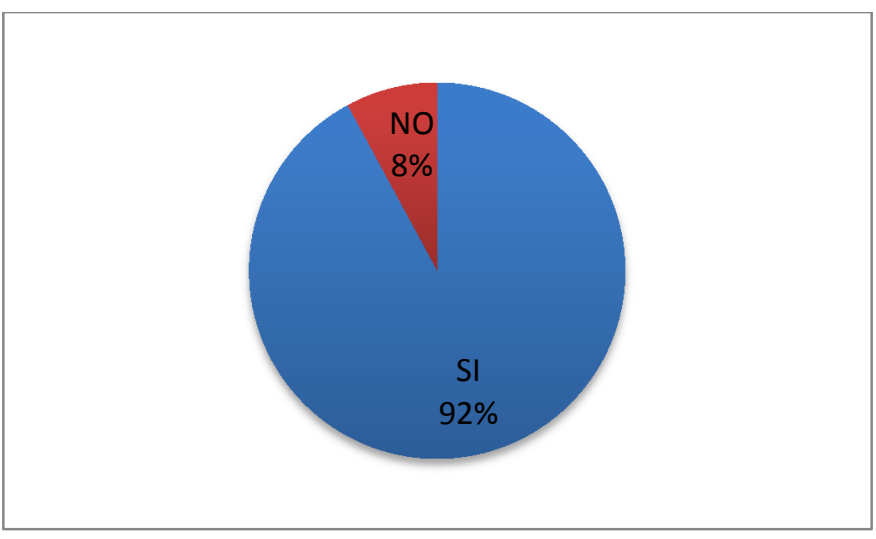

Grafica 8. Considera importante la creación de un fondo de capital de riesgo

Pregunta 8 .

¿Con la creación de un fondo de capital de riesgo cree usted que se motivara la innovación en la UPTC sede Sogamoso?

El $87 \%$ de los docentes encuestados cree que con la creación de un fondo de capital de riesgo se motivara la innovación en la UPTC-Sogamoso, de otro lado el $13 \%$ cree que la creación de un fondo de capital de riesgo para al UPTC-Sogamoso no motivara a la creación de proyectos de base tecnológica e innovadores, Grafica 9.

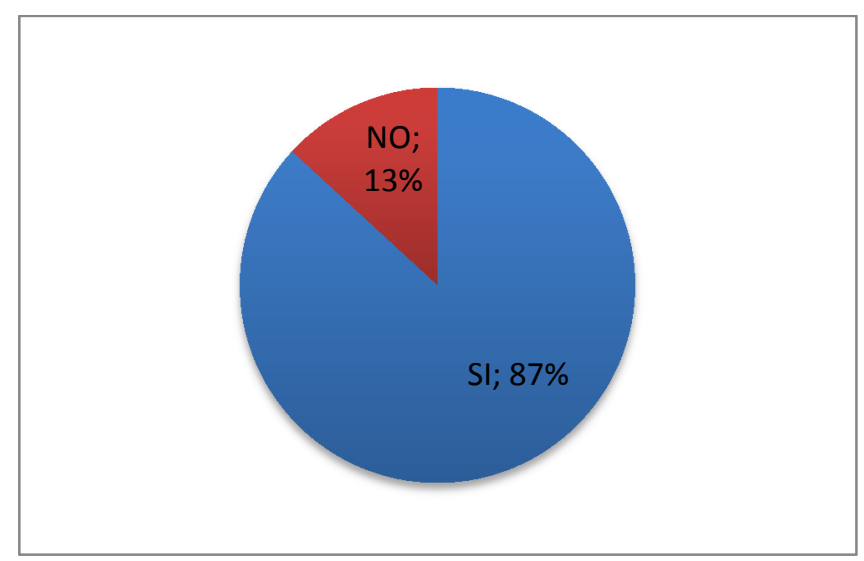

Grafica 9. Creación fondo de capital de riesgo motivara a la innovación

Pregunta 9

¿Cuál cree usted es la principal desventaja de crear empresa en Colombia?

La incertidumbre en los resultados con un $45 \%$ es la principal desventaja de crear empresa en Colombia según los docentes de la UPTC-Sogamoso, seguido de la tramitología con un $30 \%$, altamente riesgoso con el $14 \%$ y se requiere mucho 
esfuerzo con un $11 \%$ respectivamente, Grafica 10.

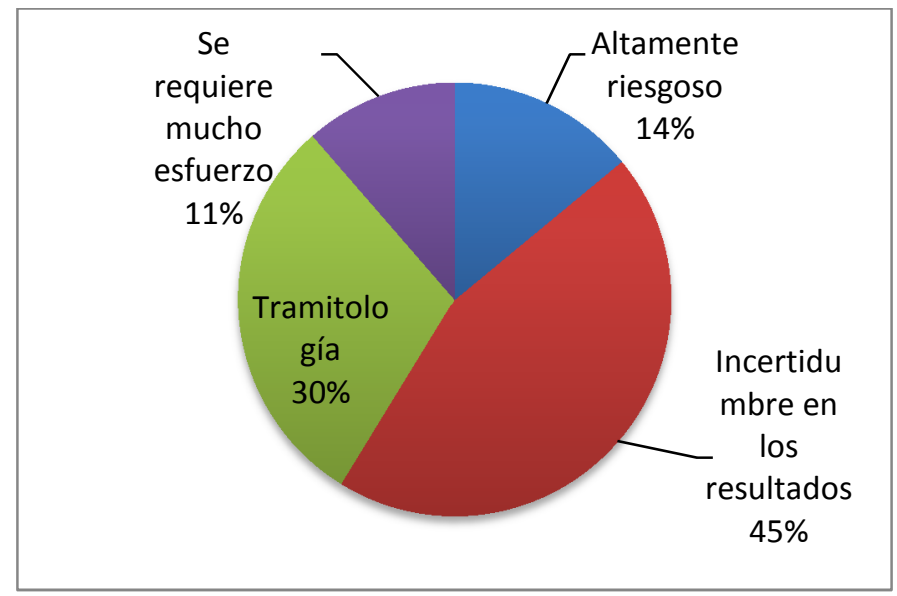

Grafica 10. Principal desventaja de crear empresa en Colombia

Pregunta 10

¿Es usted tolerante a la frustración y a la ambigüedad?

El $61 \%$ de los docentes manifiesta ser tolerantes a la frustración y a la ambigüedad y el $39 \%$ dice no serlo. Se evidencia en los docentes de la UPTC-Sogamoso una baja percepción al riesgo lo que hace que tengan una gran vocación al emprendimiento.

\section{CONCLUSIONES}

Actualmente se están desarrollando spin-off universitarios en la UPTC-Sogamoso lo cual permite crear y mantener una ventaja competitiva con respecto a las demás sedes de la Universidad Pedagógica y Tecnológica de Colombia y demás universidades del país.

El acceso a la financiación parece ser una restricción relevante para el desarrollo y crecimiento de los proyectos de base tecnológica e innovadores que se generan en los laboratorios de la UPTC-Sogamoso, es más, a la financiación con capital de riesgo piensa acceder el $56 \%$ mientras que el $40 \%$ piensa apalancar sus proyectos con recursos propios y el $4 \%$ mediante crédito bancario.

Para el 92\% de los docentes es importante la creación de un fondo de capital de riesgo para la UPTC-Sogamoso de igual manera el $87 \%$ de los docentes cree que con la creación del fondo se motivara la innovación en la sede.

Es importante resaltar el grado de percepción al riesgo de los docentes de la UPTC-Sogamoso, el 61\% de los docentes manifiestan ser tolerantes a la frustración y a la ambigüedad y el $39 \%$ dice no serlo. Estos resultados permiten afirmar que los docentes de la sede tienen una baja percepción al riesgo lo que hace que tengan una gran vocación al emprendimiento.

Las economías occidentales dedican muchos recursos públicos para favorecer la creación de nuevas empresas y superar el llamado fracaso del mercado de la innovación, es interesante centrarse en las universidades, entidades especializadas en la generación de conocimiento y por lo tanto donde potencialmente puede haber un porcentaje más alto de emprendedores innovadores, el presente estudio de investigación de mercados evidencia la factibilidad para la creación de un fondo de capital de riesgo para la UPTC sede Sogamoso.

\section{REFERENCIAS}

[1]. D. Kenney, D, Hsu “Organizing venture capital," Oxford journals, Economics, August 2005, pp 3-7.

[2]. W. Bygrave, J, Timmons. "Venture Capital. Boston," Harvard Business School Press, 1992.

[3]. W. Bygrave, J, Timmons. "Venture Capital. Boston," Harvard Business School Press, 1992.

[4]. P. Kotler, G, Arsmstrong, "Marketing versión para Latinoamérica", 13 ed., ed. México: Pearson, 2008, pp. 109123.

[5]. C. Martínez, "Estadística y muestro", 12 ed., ed. Colombia: Ecoe ediciones, 2005, pp. 389-411. 